\title{
Diseño e implementación de una plataforma digital para los proyectos de ingeniería en la empresa Niza Ingeniería S.A.S.
}

\author{
Eliecer Almario Fajardo; Isaac Penso Atencia \& José Ledesma León \\ Programa de Ingeniería Electrónica y Telecomunicaciones, Universidad Autónoma del Caribe, Barranquilla, \\ Colombia. \\ eliecer.almario@uac.edu.co, isaac.penso@uac.edu.co,jose.ledesma@uac.edu.co
}

Recibido: Octubre 15, 2020.

Recibido en su versión corregida: Enero 08, 2021.

Aceptación: Marzo 12, 2021.

https://doi.org/10.54606/Sextante2021.v24.05

Cómo citar: Almario Fajardo, E., Penso Atencia, I. \& Ledesma León, J. (2021) Diseño e implementación de una plataforma digital para los proyectos de ingeniería en la empresa Niza Ingeniería S.A.S. Revista Sextante, 24, pp. 34 - 43, 2021.

\section{Resumen}

En el presente artículo se expone el proceso de implementación del proyecto consistente en la adecuación de tecnologías digitales para desarrollar una herramienta de servicio a la empresa NIZA Ingeniería S.A.S. El proyecto buscaba, principalmente, que la compañía se desmarcara de la competencia, ofreciendo a sus clientes una plataforma digital que le permitiera estar al tanto del estado y avances de los proyectos contratados con la misma. Para acceder a la plataforma diseñada se requiere únicamente tener acceso a Internet y algún dispositivo que permita el acceso al mismo, tales como: smartphones, tabletas u ordenadores. Para ingresar a la ventana de usuario cada cliente de la empresa contará con un usuario y contraseña suministrados por la misma. Dentro de la plataforma se podrá encontrar un listado de cada proyecto contratado por el cliente. Cada proyecto contiene la información pertinente del mismo, como son: el tiempo estipulado de ejecución, personal de la empresa encargado o los trabajos específicos a realizar. Se podrán descargar archivos relacionados al proyecto como la orden de compra o la factura asociada y además una pestaña para contenido audiovisual que contienen imágenes tomadas en el transcurso de las actividades del proyecto que permitan dar evidencia de los avances que se tienen. El desarrollo de la plataforma se dividió en cuatro secciones: diseño de la interfaz de usuario, FrontEnd, BackEnd y despliegue. Se implementaron las tecnologías más usadas en la comunidad de desarrolladores como fueron: PyCharm, como editor de código; Adobe XD, para el diseño; HTML y CSS, para FrontEnd; y MySQL, para la base de datos en el BackEnd. Finalmente se presentó la plataforma a los representantes de la empresa y se realizaron pruebas con proyectos en ejecución para verificar el funcionamiento de la aplicación.

Palabras clave: Diseño de plataforma digital web; Gestión de proyectos; Indicadores de rendimiento; Innovación en proyectos.

\section{Design and implementation of a digital platform for engineering projects in the company} NiZA Ingeniería S.A.S.

\begin{abstract}
This article describes the project's implementation process consisting of digital technologies to develop a tool for the company NIZA Ingeniería S.A.S. The project sought mainly for the company to stand out from the competition, offering its customers a digital platform that would allow them to be aware of the status and progress of the projects contracted with the company. The access of the designed platform was only required to have internet access and a device that allowed access to it, such as smartphones, tablets, or computers. To enter the user window, each client of the company will have a username and password provided by the company. Within the platform, it can find a list of each project contracted by the client, and each project contains the relevant information of the same, such as the stipulated time of execution, company personnel in charge, or the specific work to be performed. Files related to the project can be downloaded, such as the purchase order or the associated invoice, and a tab for audiovisual content containing images taken during the project's activities, evidence of the progress made. The platform's development was divided into four sections: user interface design, FrontEnd, BackEnd, and deployment. The most used technologies in the developer community were implemented: PyCharm as a code editor, Adobe XD for the design, HTML, and CSS for the FrontEnd and MySQL for the database in the BackEnd. Finally, the platform was presented to the company's representatives, and tests were carried out with projects in execution to verify the application's performance.
\end{abstract}

Keywords: Innovation in projects; Performance indicators; Project management; Web digital platform design. 


\section{Introducción}

Actualmente las tecnologías están en constante desarrollo, en consecuencia, algo tan trivial como saber el clima o conocer el estado de cierta variable de una ciudad era algo muy poco viable varias décadas atrás, y es a causa de estas facilidades que cada día a las personas les es más fácil tener conocimiento del estado de su entorno de una manera más eficaz y precisa.

En vista de lo anteriormente expuesto, trasladándolo al ámbito empresarial, el objetivo de todas las empresas siempre estará enfocado en encontrar el método para lograr el mejor posicionamiento comparado con su competencia, permitiendo así, llegar a más personas y clientes y, por ende, tener un día a día rentable por medio del balance de sus ganancias. Por otro lado, en su búsqueda de lograr el mayor posicionamiento posible es normal que las compañías se topen con las tecnologías que les permitan automatizar y monitorear procesos en pro de reducir sus gastos operativos y a su vez optimizarlos; una vez hecho esto, confiando en la exactitud alcanzada por una maquina en un proceso específico, se puede concluir que el resultado será mucho más completo en cuanto estándares de calidad, repercutiendo positivamente en el nombre de la empresa, la cual elabora algún producto en particular o brinda algún servicio, ocasionando así, que la imagen de dicha empresa sea mucho más seductora para los potenciales clientes y usuarios de esta.

Entrando en contexto, la empresa NIZA Ingeniería S.A.S es una empresa caracterizada por destacarse en el sector de atención al cliente y excelente prestación de servicios, con estándares de calidad competitivos, relacionados con proyectos de ingeniería en las áreas civil, HVAC e instalaciones eléctricas. Sin embargo, el reto constante que esta presenta es el permanecer en una continua evolución para no ser reducida por las demás compañías que conforman la competencia, ya que con bien se conoce, en esto radica el éxito de cualquier empresa. Con miras de lograr esta incesante mejora, el objetivo de este trabajo es crear un factor diferencial que le permita desmarcarse de las demás empresas de su sector, ya que normalmente en proyectos con empresas pequeñas o medianas los clientes no tienen ningún medio con el cual monitorear, observar o mantenerse al tanto de los avances y progresos de los trabajos.

Es por eso por lo que a partir de la ejecución de este trabajo se espera que la empresa Niza Ingeniería S.A.S. alcance niveles de competitividad altos para los clientes en el área de proyectos de ingeniería, ofreciendo una plataforma digital que pueda mejorar la calidad de los servicios. De la misma manera se busca mejorar el impacto ambiental en dichos proyectos, debido a que la plataforma al ser una herramienta digital no involucraría el uso de nuevos recursos físicos ni materias primas adicionales necesarias para su desarrollo, elaboración e implementación en el sector empresarial.

\section{Estado del arte}

A nivel nacional han germinado diversos proyectos enfocados en esta área de innovación y mejoras de procesos, los cuales en su mayoría se apoyan en el monitoreo de variables de un proceso en específico, las cuales son remitidas posteriormente según el formato y emisario que se desee, ya sea a un cliente o a la misma empresa que esté haciendo uso de la herramienta software. Esto con la intención de incrementar la calidad del servicio prestado y en caso de que ocurra algún inconveniente, lograr tomar acciones correctivas en el menor lapso posible.

Es el caso del proyecto llevado a cabo por Gonzalez Avila (2014), consistente en una red de monitoreo por medio del uso del protocolo SNMP para la gestión y monitoreo de La Red Nacional Académica de Tecnología Avanzada (RENATA) (¿Qué Es RENATA? - Red RENATA, n.d.) la cual es la red nacional de investigación y educación de Colombia que permite la comunicación entre diferentes entidades a nivel nacional e internacional.

Por otro lado, el proyecto realizado por Huayhuapuma Jara \& Moriano Marca (2018) de la Universidad Autónoma de Perú consistía en un software capaz de monitorear el estado de las puertas automáticas en la empresa MARKHA SECURITY S.A.C a través de una interfaz web. Para lograr este objetivo usaron las plataformas ARDUINO y UBIDOTS (Ubidots । ConnectAmericas, n.d.), las cuales les permitieron enviar datos de sensores a la nube, configurar 
tableros y alertas, conectarse con otras plataformas, usar herramientas de analítica y arrojar mapas de datos en tiempo real.

En esa misma línea de monitorización, el trabajo elaborado por Asencio Vargas (2017), consiste en una aplicación móvil, así como su contraparte web, que facultan el monitoreo por geolocalización de unidades de transportes de carga pesada para la empresa TRANSPORTES Y LOGISTICA HONORES MENDEZ en Ecuador. Esto se logra por medio de un GPS instalado en cada uno de los transportes de carga pesada, de los cuales se recolecta la información de la posición, para posteriormente, dicha información, ser trasladada a un servidor en el que estos datos se recolectarán, y así ser analizados por el usuario a través de una interfaz web o también por medio de una aplicación móvil dedicada para ello.

Existen diversas empresas encargadas de brindar el servicio de monitoreo en tiempo real de proyectos industriales. A nivel nacional tenemos a la empresa TELEMETRIK (Automatización Industrial, Monitoreo y Control de Procesos Industriales y de Producción / Internet de Las Cosas (IoT) / Teleprocess, n.d.), que junto con su hardware llamado Teleprocesos son capaces de medir en tiempo real, por medio de una plataforma, cualquier variable física dentro de máquinas o equipos activos durante procesos industriales, para determinar el estado de los mismos y en caso de presentarse escenarios inesperados, generar alertas por medio de mensajes de texto dirigidos a los celulares de los usuarios o a sus correos electrónicos, con el objetivo de preparar una toma de decisiones lo más rápida posible y evitar fallos que afecten el desempeño de la producción.

En esta misma orientación se encuentra la empresa BISMARK (Manufactura - Bismark Colombia, n.d.), localizada en la ciudad de Bogotá, con más de dos décadas en el mercado. Esta se encarga, principalmente, del asesoramiento e implementación de Industria 4.0. Gracias a esto sus clientes pueden saber en todo momento el estado de su maquinaria y de los procesos que se están ejecutando para la toma oportuna de decisiones en caso de que algún contratiempo se presente.

A nivel internacional se encuentra el GRUPO GARATU (Plataforma IoT Industrial, Monitoreo y
Control de Sistemas, n.d.), que brinda un sistema de monitoreo de proyectos alrededor de diversos países de Europa y Latinoamérica por medio de su plataforma IOT, la cual se ajusta dependiendo de las necesidades que el proyecto requiera. También podemos encontrar a la empresa AMAZON, que de por sí cuenta con un servicio de recolección de datos públicos como es Amazon Web Service, el cual permite la recolección de datos de las diferentes maquinarias, posibilitando que dicha maquinaria pueda ser monitoreada y con esto saber el estado de estas y los procesos que realizan diariamente. Este tipo de servicio se realiza dependiendo de las necesidades que tenga la empresa y en caso de que el cliente no sepa con exactitud lo que requiere para el proyecto, AMAZON colabora brindando ciertas directrices acerca de los potenciales requerimientos para la exitosa implementación del proyecto de la empresa contratante.

\section{Metodología propuesta}

\subsection{Requerimientos necesarios}

Previo a mostrar el desarrollo metodológico seguido para la creación e implementación de la herramienta, es necesario exponer los requisitos mínimos necesarios para su puesta en marcha, tanto en el punto de vista técnico como en el de planeación de actividades y caracterización de esta.

En el marco de desarrollo técnico se debieron cumplir los siguientes requisitos:

\section{Para el desarrollo de la plataforma se requieren} los siguientes programas o herramientas:

- Lenguaje de programación: Phyton 3.8.

- Editor de código: PyCharm.

- Diseño del prototipo no funcional de la plataforma: Adobe XD.

- Interacción con el navegador: HTML, CCS, JavaScript y Bootstrap 4.

- Dominio GoDaddy y registros DNS en Amazon web services.

\section{Requisitos del sistema recomendados}

- Sistema operativo Windows 10 (o cualquiera compatible con el editor de código Pycharm).

- Mínimo 500MB de RAM.

- Procesador Intel ${ }^{\circledR}$ Core $^{\mathrm{TM}} \mathrm{i} 3$ o superior. 
- Mínimo 20 GB de almacenamiento.

\section{Requerimientos para el usuario}

- Dispositivo electrónico con acceso a Internet: Computador, tablet o smartphone.

En el marco del desarrollo y la planeación de la plataforma a crear, esta deberá cumplir los siguientes requerimientos:

- Acceso desde dispositivos de escritorio o móviles.

- La plataforma debe contar con un sistema de seguridad basado en usuario y contraseña.

- En la plataforma los usuarios deberán encontrar un listado de los proyectos contratados con Niza Ingeniería SAS. Se debe visualizar información relevante a este (nombre del proyecto, información del personal encargado, tiempo estipulado, trabajos a realizar), además, archivos descargables como la cotización o la orden de servicio.
- Deberá disponer de contenido multimedia descargable para complementar la información del proyecto.

- Deberá contar con un sistema de calificación, en donde el usuario dejará su información personal y su calificación, para su posterior almacenamiento y análisis.

\subsection{Ejecución metodológica}

En la Figura 1, se observa el diagrama con las herramientas utilizadas en la creación de la plataforma y su respectiva línea de desarrollo, para entender el relacionamiento entre estas y el orden en que fueron aplicadas.

Como se observa en la Figura 1, para el desarrollo del framework web se utilizaron las herramientas de Django y el editor de código Pycharm, ambos seleccionados por su facilidad de adaptabilidad por parte del usuario, gracias a sus numerosos paquetes, herramientas y ayudas que ponen al servicio de los programadores, para poder hacer mucho más amena la experiencia de edición de código.

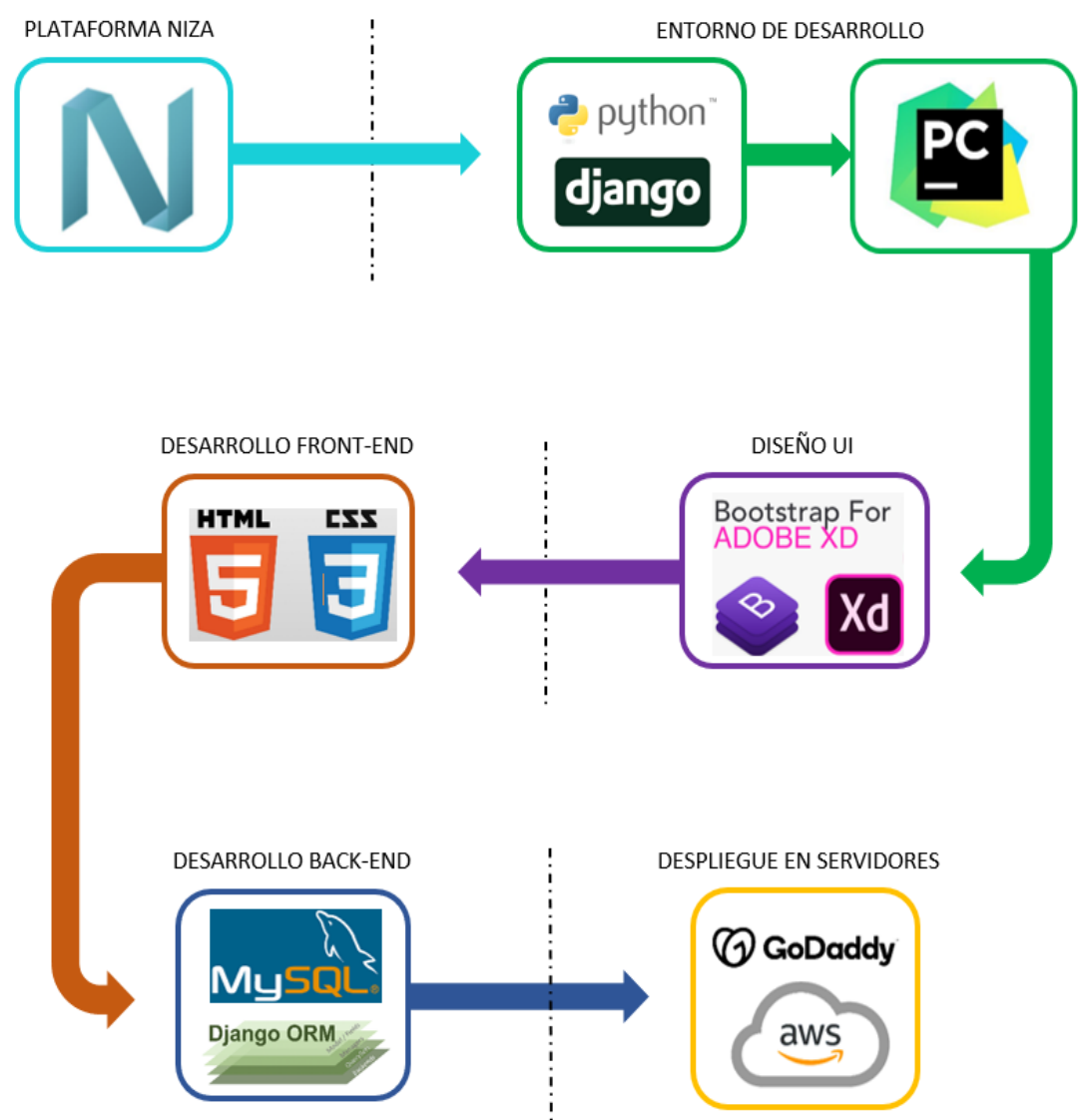

Figura 1. Gráfico de las herramientas utilizadas para la realización de la plataforma.

Fuente: Los autores. 
Seguidamente, para el diseño del prototipo de la interfaz de usuario se decidió utilizar el programa Adobe $\mathrm{XD}$, debido a su potencia en proyectos relacionados a diseño de experiencias e interfaces de usuario, tanto para sitios web como para aplicaciones móviles. También se hizo uso del kit Material Design de Bootstrap 4, uno de los kits de herramientas de código abierto de front-end más destacados en el mundo de la programación, el cual ofrece amplios componentes prediseñados $\mathrm{y}$ potentes complementos de JavaScript. Lo anteriormente mencionado se ejecutó siguiendo la metodología de desarrollo de aplicaciones mobile First. Con el objetivo de ir aumentando progresivamente el escalado y el tamaño de los objetos dispuestos en la interfaz de usuario hasta llegar a las dimensiones de una pantalla de escritorio.

El kit de Material Design se puede encontrar en el siguiente enlace web: https://www.w3schools.com/bootstrap4/bootstrap ref_js_button.asp. En la Figura 2, se observa el diseño de la plataforma en su primera versión enfocada a dispositivos móviles.

En cuanto al desarrollo del frontend integró con HTML $\mathrm{y}$

CSS (https://www.w3schools.com/bootstrap4/bootstrap ref_js_button.asp) los componentes visuales diseñados previamente en Adobe XD para que el usuario final interactuara con ellos en el navegador.

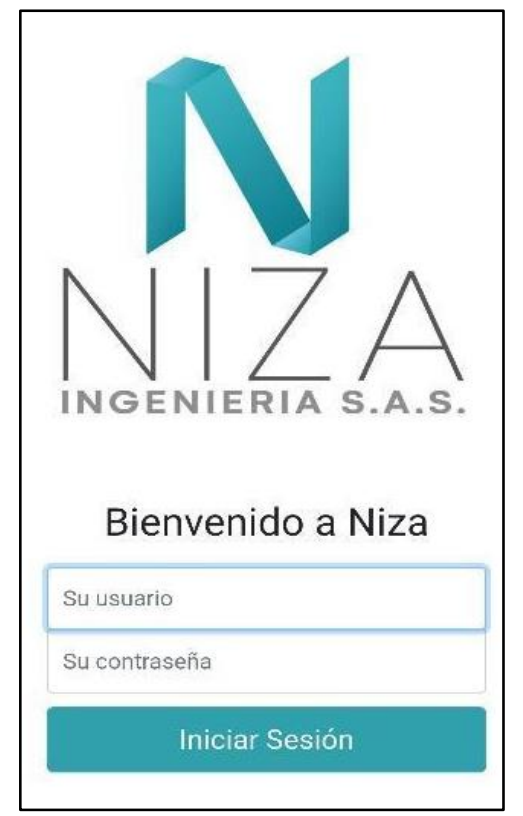

Figura 2. Diseños de la plataforma en su versión para dispositivos móviles. Fuente: Los autores.
Posteriormente, no olvidando la función integrada para obtener la calificación de la plataforma, se utilizó una librería llamada sweet alert 2, que tiene como principal objetivo notificar al usuario cuando se envía correctamente una calificación de la plataforma. Esta librería se puede encontrar en el siguiente enlace: https://sweetalert2.github.io/

Por último, en cuanto al backend se diseñó una base de datos de tipo relacional (ver Figura 3), la cual se encargará de almacenar y proporcionar acceso a puntos de datos relacionados entre sí. El motivo por el cual se seleccionaron las bases de datos relacionales es debido a que estas se ajustan a las necesidades de la plataforma, ya que se utilizan para hacer seguimiento de los inventarios, administrar grandes cantidades de información de clientes de misión crítica y mucho más.

Para el sistema de gestión de bases de datos de tipo relacional se empleó el programa MySQL de la empresa Oracle Corporation, debido a que es considerada como la base de datos de código abierto más popular del mundo.

Para efectos de simplificar el desarrollo de la base de datos se utilizó una capa de mapeo relacional de objetos (ORM) predeterminada, que se puede usar para interactuar con datos de aplicaciones de varias bases de datos relacionales como MySQL. Esto está incluido en el framework de Django.

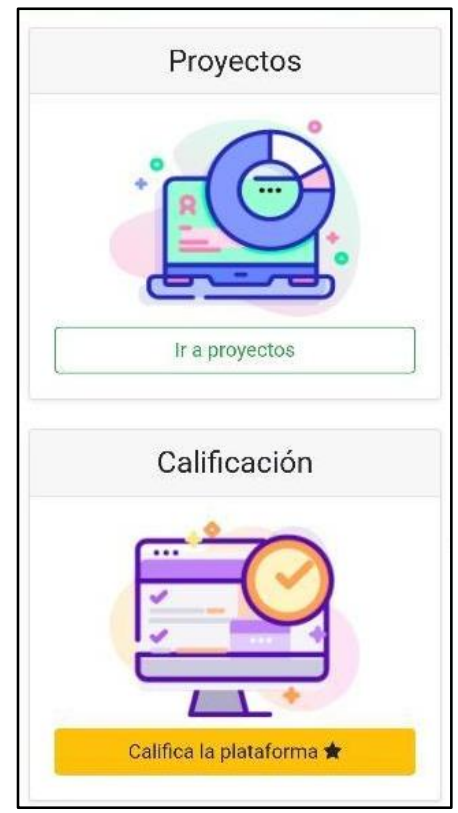




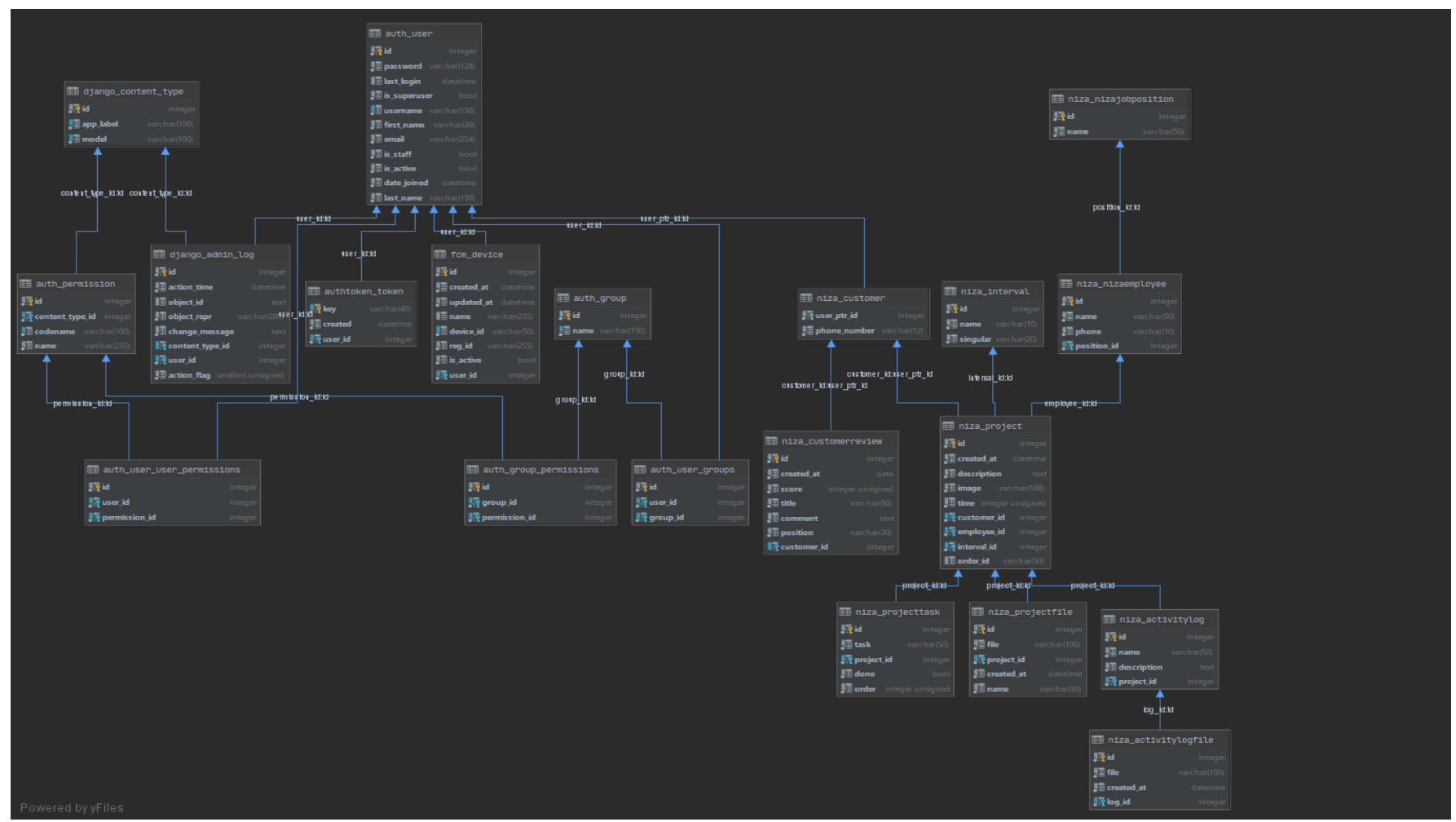

Figura 3. Esquema relacional de la base de datos.

Fuente: Los autores.

Utilizando ORM de Django se lograron crear las tablas de la base de datos para así evitar escribir código SQL (que puede llegar a ser complejo para programadores inexpertos).

Para hacer cambios en la base de datos, se utilizó el siguiente modelo de migraciones:

- Ejecutar python manage.py makemigrations para crear migraciones de esos cambios.

- Ejecutar python manage.py migrate para aplicar esos cambios a la base de datos.

Las clases que conforman la arquitectura de la base de datos son las siguientes:

- Customer: en donde se asignará tipo de usuario, nombre y contraseña.

- Projects: en donde se crearán los proyectos de los usuarios.

- Project Task: en donde se abdicarán los trabajos específicos a realizar en cada proyecto.

- Intervals: en donde se indicará la duración del proyecto en días, semanas o meses.
- Niza Employee: en donde se indicará la información del personal de la empresa encargado de la ejecución del proyecto.

- Niza Job Position: en donde se indicará el puesto del personal encargado de la ejecución del proyecto.

- Activities Logs: en donde se registran las actividades, hechos, avances o incidencias relacionadas con los trabajos específicos de cada proyecto. Esto se hará de forma periódica, coincidiendo con la duración del proyecto.

- Customer Reviews: en donde se almacenará la calificación y los comentarios de los usuarios respecto a la plataforma.

\subsection{Configuración de infraestructura y despliegue}

Debido a que existe la imperativa necesidad de que la plataforma funcione desde cualquier parte del mundo y en todos los dispositivos móviles y de escritorio, fue necesario subir la plataforma en un servidor en la nube. (En esta etapa se usaron los recursos que la empresa Niza Ingeniería SAS dispuso para el proyecto, como los servicios ya 
contratados con AWS (Amazon Web Services), y el personal encargado de manejar estos servicios).

Se prefirió a AWS (ver Figura 4), como proveedor de infraestructura a través del servicio $\mathrm{EC} 2$, el cual permite crear máquinas virtuales (servidores), con cualquier distribución de Linux y/o Windows (aunque en este caso se utilizó Ubuntu server 18.10), y se pueda crear una conexión a estas máquinas por medio del protocolo SSH usando un archivo con extensión .pem como llave de entrada al servidor, el host y el usuario que el servidor crea por defecto.

Posteriormente se seleccionó un servidor, una instancia de tipo t2.micro. En la instancia se instalaron todas librerías que requiere el proyecto para funcionar. Además, se instaló UWSGI, NGINX y CERTBOT para establecer el certificado SSL, el cual marca como segura la conexión de un sitio web, garantizando que la conexión es privada entre cliente y servidor.

\section{Resultados}

Se puede acceder a la plataforma NIZA desde cualquier dispositivo electrónico inteligente con conexión a Internet por medio del siguiente enlace: https://app.nizaingenieria.com/ (ver Figura 5). Se debe ingresar usuario y contraseña suministrada por Niza Ingeniera SAS. Dependiendo del tipo de usuario se podrá acceder a distintas instancias. Si el usuario es administrador, encontrarán una interfaz para configurar, agregar o eliminar contenido a la plataforma como se observa en la Figura 6.

En el caso de que el usuario sea un cliente, la interfaz será muy diferente. Como primera instancia encontrará dos opciones para seguir navegando en la plataforma: la primera es VER EL LISTADO DE PROYECTOS y la segunda es EVALUAR LA PLATAFORMA (ver Figura 7). Si se desea calificar la plataforma, el usuario tendrá que dejar sus datos, un comentario y una calificación (ver Figura 8).

Si se desea explorar los proyectos, el usuario encontrará los contratados con la empresa NIZA Ingeniería S.A.S. ya sean terminados o en ejecución (ver Figura 9).

Al entrar en un proyecto se encontrará con una sección de información general de este y una sección donde se registrarán periódicamente los trabajos realizados con evidencias fotográficas. Se podrán descargar documentos y archivos multimedia. Dentro de los proyectos el usuario encontrará todo lo relacionado con estos (ver: Figura 10).

\begin{tabular}{|c|c|c|c|c|}
\hline \multicolumn{2}{|c|}{$\begin{array}{l}\text { Resumen de instancia de i-03b021ed3bd9366c7 (niza_platform) Información } \\
\text { Se ha actualizado hace less than a minute }\end{array}$} & C & Conectar & Estado de la \\
\hline ID de la instancia & Dirección IPv4 pública & \multicolumn{3}{|c|}{ Direcciones IPv4 privadas } \\
\hline [.] i-03b021ed3bd9366c7 (niza_platform) & (1] 52.15 .175 .74 (niza_platform) | dirección abierta [ & \multicolumn{3}{|c|}{ ㄱ. 172.31 .27 .254} \\
\hline Estado de la instancia & DNS de IPv4 pública & \multicolumn{3}{|c|}{ DNS IPV4 privado } \\
\hline (-En ejecución & $\begin{array}{l}\text { ec2-52-15-175-74.us-east-2.compute.amazonaws.com | } \\
\text { dirección abierta [ }\end{array}$ & \multicolumn{3}{|c|}{ (1) ip-172-31-27-254.us-east-2.compute.internal } \\
\hline Tipo de instancia & Direcciones IP elásticas & \multicolumn{3}{|l|}{ ID de VPC } \\
\hline t2.micro & [.] 52.15.175.74 (niza_platform) [IP pública] & \multicolumn{3}{|c|}{ [.] $v p c-e f 76 b 984[$} \\
\hline Hallazgo de AWS Compute Optimizer & Rol de IAM & \multicolumn{3}{|l|}{ ID de subred } \\
\hline $\begin{array}{l}\text { (1) Suscribirse a AWS Compute Optimizer para recibir } \\
\text { recomendaciones. | Más información } 匹\end{array}$ & - & \multicolumn{3}{|c|}{ [1] subnet-b5210dcf $[\pi$} \\
\hline
\end{tabular}

Figura 4. Resumen de la instancia en Amazon Web Services.

Fuente: Los autores 


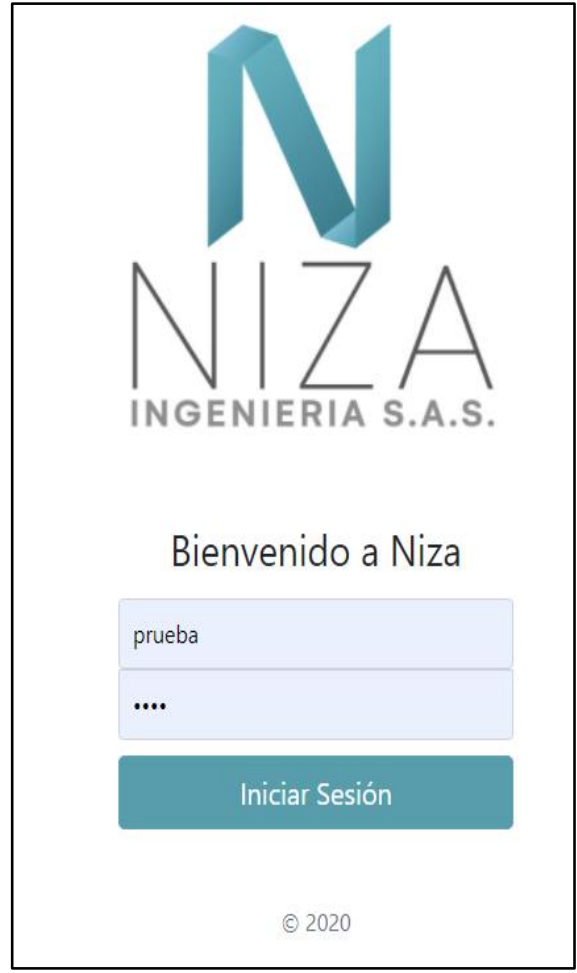

Figura 5. Login de escritorio de la plataforma.

Fuente: Los autores.

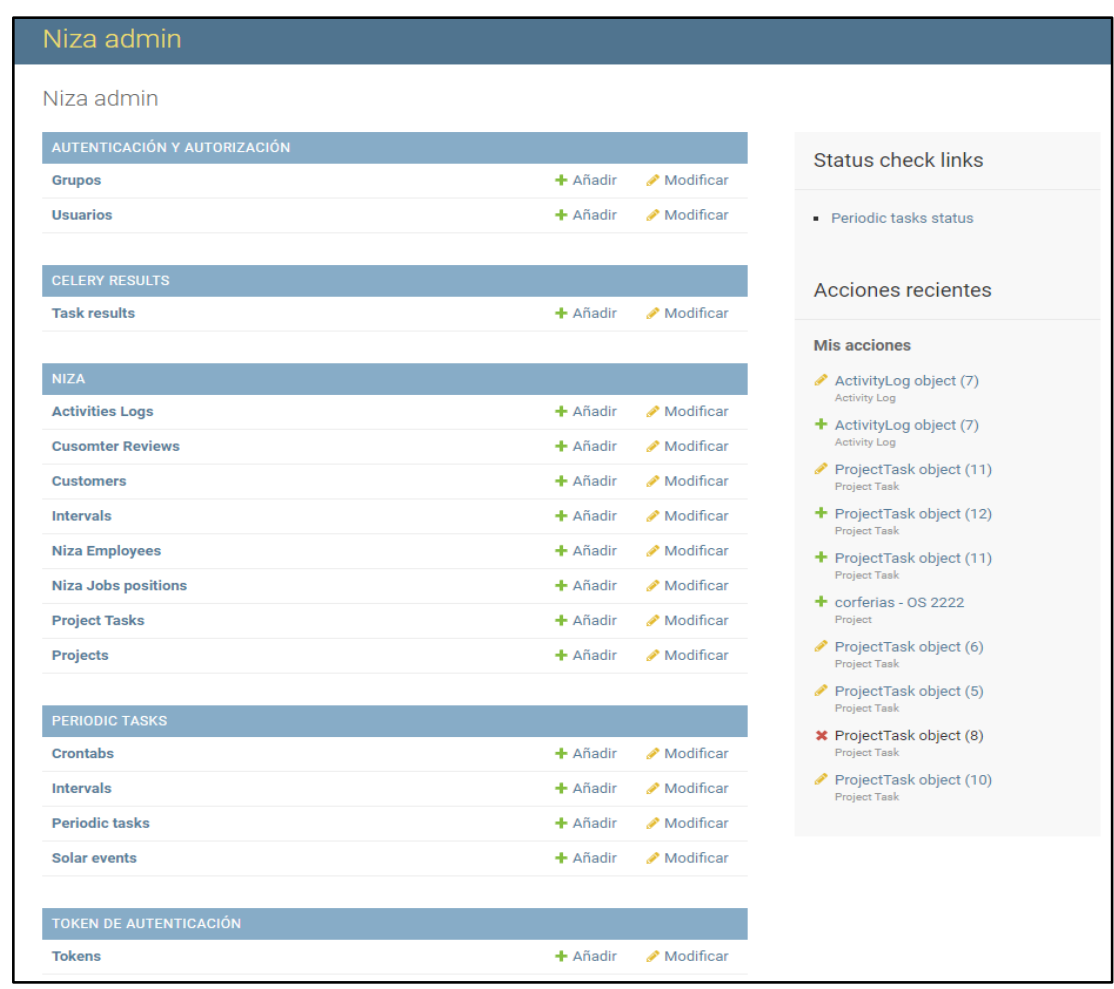

Figura 6. Interfaz para administradores.

Fuente: Los autores.

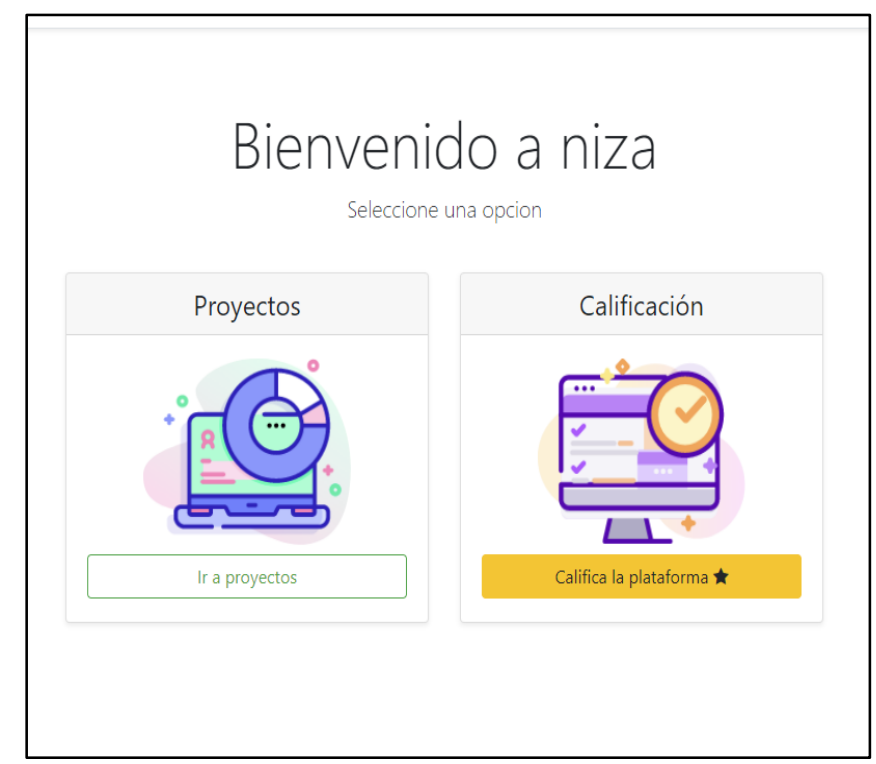

Figura 7. Interfaz de inicio para usuarios clientes.

Fuente: Los autores.

\section{Conclusiones}

Al finalizar el proyecto la presentación de la plataforma digital generó reacciones positivas $\mathrm{y}$ entusiasmo por parte de los representantes de la empresa NIZA Ingeniería S.A.S. La plataforma cumplió con las expectativas y objetivos de desarrollo planteados desde principios del proyecto. Se acreditó la plataforma con proyectos en curso

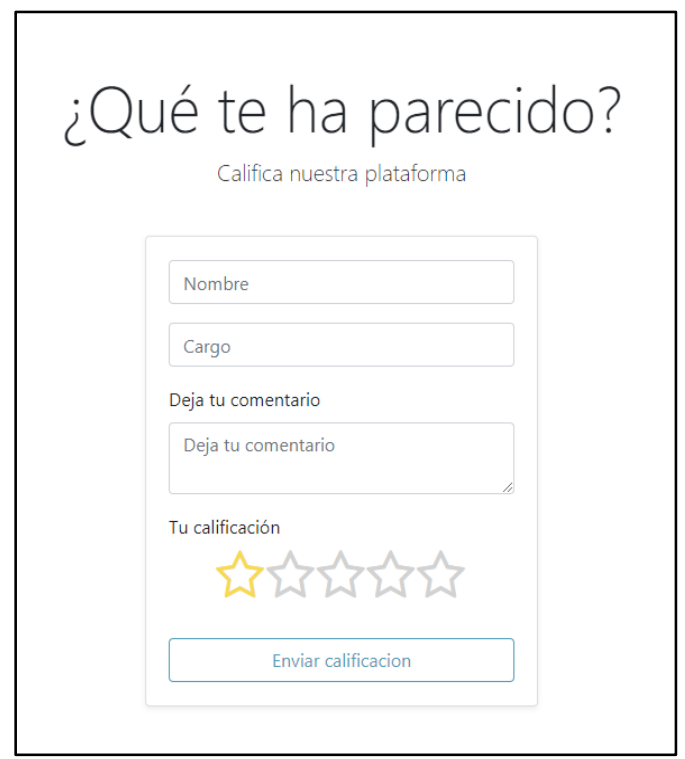

Figura 8. Interfaz de usuario para evaluar la plataforma. Fuente: Los autores.

contratados por clientes de NIZA, específicamente PUERTA DE ORO y CORFERIAS comprobando así su óptima funcionalidad. Producto del proceso de diseño y desarrollo de la plataforma se logran remarcar varios éxitos y aspectos a mejorar.

Como aspecto positivo, la elección de PyCharm como editor de código y el framework Django fue un factor determinante en el curso del proyecto ya 


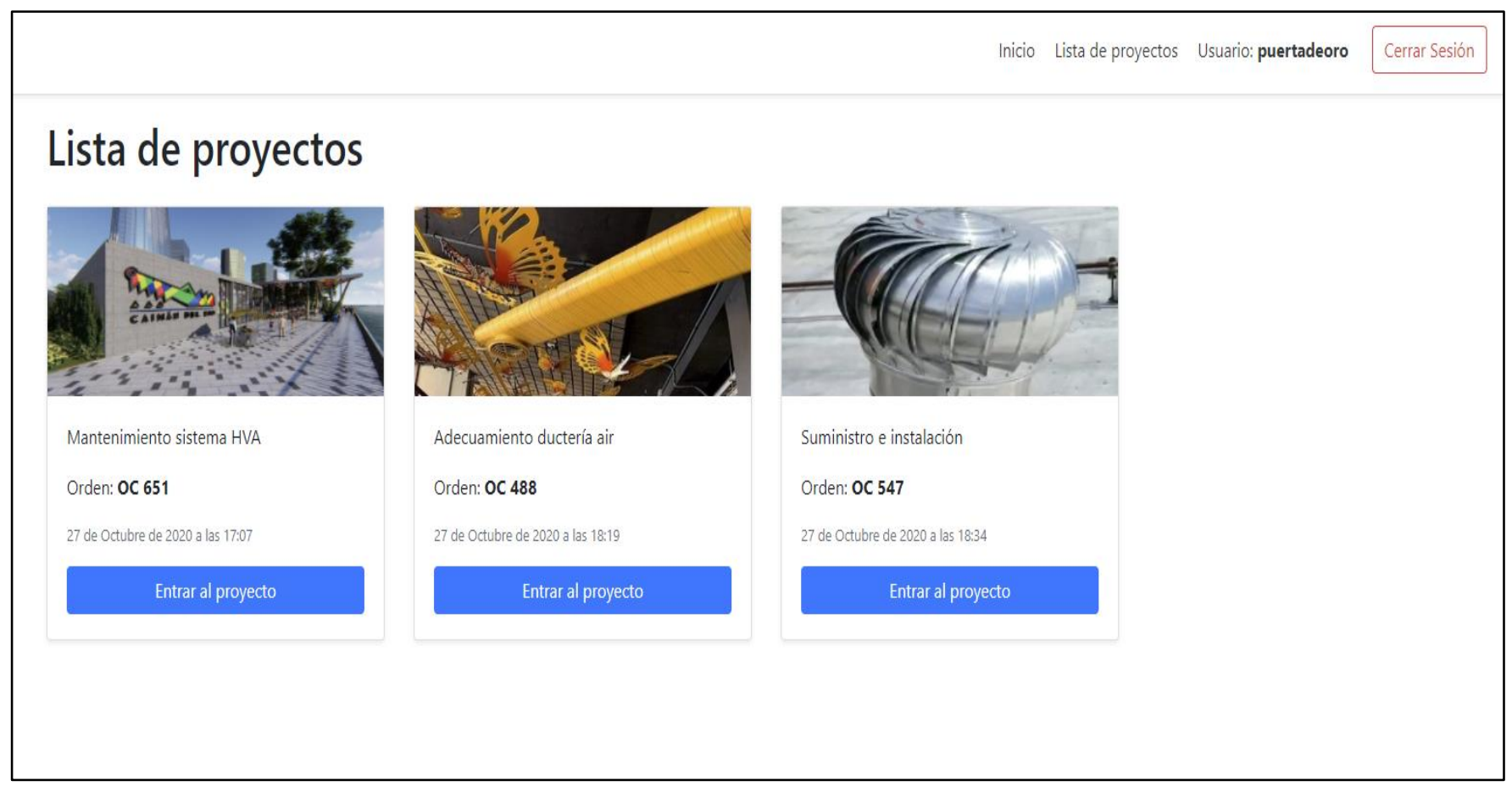

Figura 9. Interfaz de usuario para explorar y visualizar todos los proyectos contratados con NIZA Ingeniería SAS.

Fuente: Los autores.

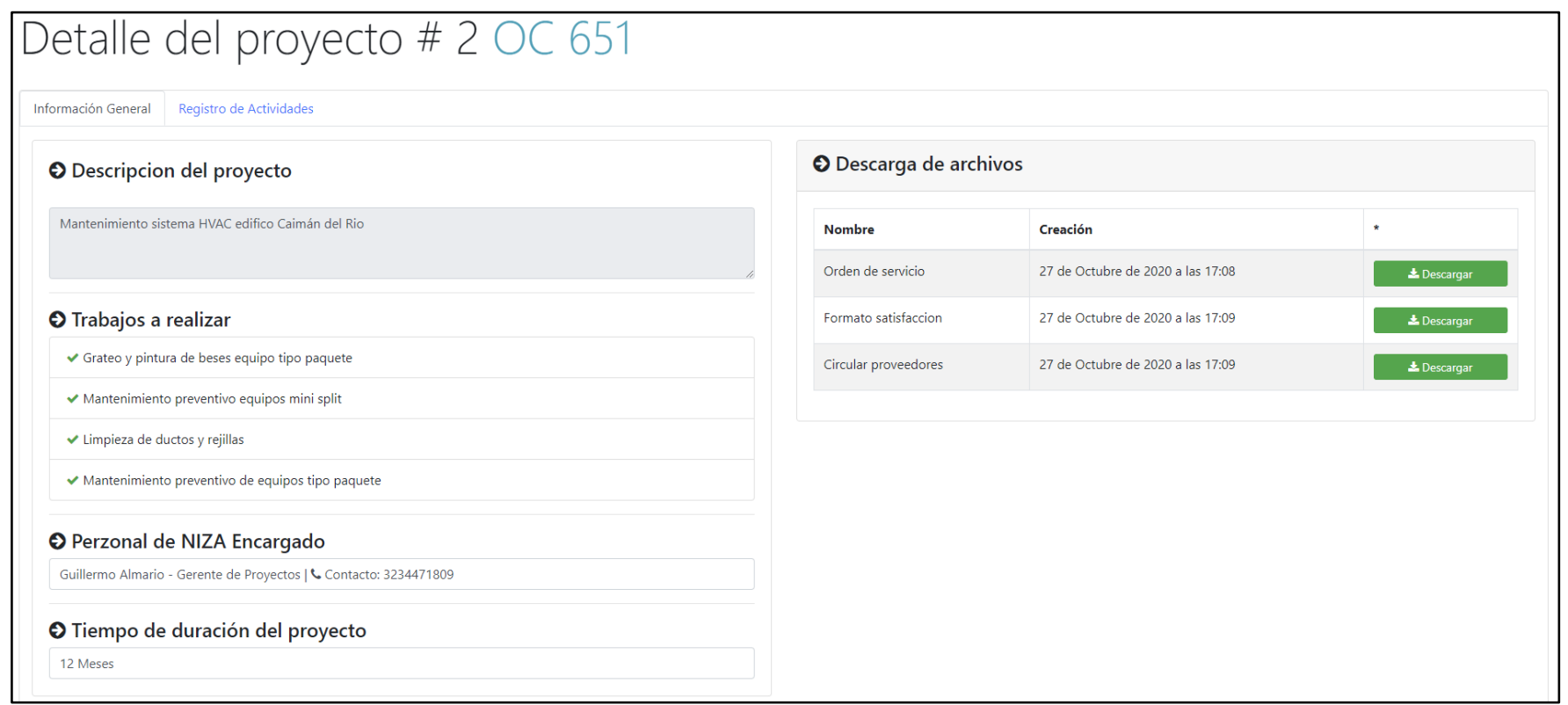

Figura 10. Sección de información y archivos generales del proyecto seleccionado en la pestaña de la Figura 9.

Fuente: Los autores.

que estos se convirtieron en herramientas cruciales para lograr los hitos mostrados en la sección de resultados: la magnífica visualización y edición de la base de datos en el mismo entorno de desarrollo; y el ORM de Django para facilitar la programación de la base de datos fueron fundamentales para la simplificación del proyecto.

Por otro lado, la configuración de la base de datos de tipo relacional arrojó resultados que cumplieron con los objetivos trazados, pero que pueden ser perfectibles. Específicamente se encontró que la estructura de la base de datos conlleva a un almacenamiento de la información que no separa las "clases" por clientes, lo que fomenta que la información no se discrimine de manera correcta. De esta forma, se pueden encontrar, en la clase "proyects", proyectos de clientes distintos mezclados entre sí, esto es debido a que la clase "customers" (que contiene a los diferentes usuarios) no se distingue por encima de las demás clases. 
Esto no se convierte en un problema grave, pero a futuro se espera optimizar la plataforma con el objetivo de jerarquizar más ordenadamente el contenido, y que no sea percibido todo en igualdad de condiciones por parte del usuario.

\section{Referencias}

¿Qué es RENATA? - Red RENATA. (n.d.). Retrieved August 18, 2021, from https://www.renata.edu.colque-es-renatal

Asencio Vargas, R. P. (2017). DESARROLLO DE UN SISTEMA WEB Y APLICATIVO ANDROID PARA EL CONTROL Y MONITOREO POR GEOLOCALIZACIÓN DE UNIDADES DE TRANSPORTACIÓN DE CARGA PESADA PARA LA EMPRESA TRANSPORTE Y LOGÍSTICA HONORES MÉNDEZ [UNIVERSIDAD DE GUAYAQUIL]. http://repositorio.ug.edu.ec/bitstream/redug/194 96/1/B-CISC-PTG-1237-Asencio Vargas Richard Paolo.pdf

Automatización industrial, monitoreo y control de procesos industriales y de producción / Internet de las Cosas (IoT) / Teleprocess. (n.d.). Retrieved August 18, 2021, from https://telemetrik.co/internet-de-las-cosas-iotautomatizacion-industrial-instrumentacionmonitoreo-de-procesos-industriales-control-deprocesos-industriales/

GONZALEZ AVILA, V. R. (2014). DISEÑO E IMPLEMENTACIÓN DE UN SISTEMA DE MONITOREO BASADO EN SNMP PARA LA RED NACIONAL ACAEMICA DE TECNILOGÍA AVANZADA [UNIVERSIDAD SANTO TOMAS]. https://repository.usta.edu.co/bitstream/handle/ 11634/766/disenoeimplementaciondeunsistemad emonitoreobasadoensnmpparalarednacionalaca demicadetecnologiaavanzada.pdf? sequence $=1$ \&isAllowed $=y$

HUAYHUAPUMA JARA, N. R., \& MORIANO MARCA, J. L. (2018). IMPLEMENTACIÓN DE UN SISTEMA DE MONITOREO WEB PARA MEJORAR EL SERVICIO DE MANTENIMIENTO DE PUERTAS AUTOMÁTICAS EN LA EMPRESA MARKHA SECURITY S.A.C [Universidad Autónoma del
Perú]. http://repositorio.autonoma.edu.pe/bitstream/A UTONOMA/530/1/HuayhuapumaJara\%2CNery RocxaliayMorianoMarca\%2CJoseLuis.pdf

Manufactura - Bismark Colombia. (n.d.). Retrieved August 18, 2021, from https://bismark.net.co/industrias/manufactural

Plataforma IoT industrial, monitoreo y control de sistemas. (n.d.). Retrieved August 18, 2021, from https://development.grupogaratu.com/plataform a-iot-grupo-garatu/

Ubidots | ConnectAmericas. (n.d.). Retrieved August 18, 2021, from https://connectamericas.com/es/company/ubidot $\underline{s}$ 